\title{
Shock-Formation Down a Non-Uniform Tube in Two Phase Flow
}

\author{
Kanti Pandey ${ }^{1} \&$ Preeti Verma ${ }^{1}$ \\ ${ }^{1}$ Department of Mathematics \& Astronomy, Lucknow University, Lucknow, India \\ Correspondence: Kanti Pandey, Department of Mathematics \& Astronomy, Lucknow University, Lucknow 226007, \\ India.
}

Received: July 22, 2012 Accepted: August 8, 2012 Online Published: June 28, 2013

doi:10.5539/jmr.v5n3p17 URL: http://dx.doi.org/10.5539/jmr.v5n3p17

\begin{abstract}
In present paper an attempt is made to study the one dimensional formulation of flow in a tube of varying crosssectional area for two phase flow when equilibrium is established eventually and particle volume fraction is taken as one of the additional variable. Conservation laws and shock condition are obtained and using Whitham rule (1974) of characteristic a relation between cross- sectional area and particle volume fraction is obtained and result is discussed for different values of Mach number.
\end{abstract}

Keywords: shock-formation, non-uniform tube, two-phase

Subject Classification: 76 L.T.

\section{Introduction}

Flow in a pipe or artery are certain examples of flow in non-uniform tube. Shock waves propagating in a duct of constant cross sectional area represent a particularly good example of relaxation in a gas particle mixture. If a shock wave travels through a stationary equilibrium mixture, the gas temperature and pressure undergo a practically instantaneous rise at the shock front, while the velocity decreases behind the shock front. The particles thus suddenly find themselves in a gas of different velocity and temperature until their subsequent interaction with the gas gradually establishes new equilibrium conditions.

The study of wave propagation in a mixture of gas and dust particles has received great attention during the last several decades. There are many engineering applications for flow of a medium that consists of a suspension of powdered material or liquid droplets in a gas. The mathematical analysis of such two phase flows is considerably more difficult than that of pure gas flows, and one of the usual simplifying assumptions is that the volume occupied by the particles can be neglected. In many important cases, the particles represent less than one half of the mass of the gas particle mixture, and the density of the particle material is more than a thousand times larger than the gas density. Under such conditions, the particle volume fraction is of the order of $10^{-4}$ only and the assumption of a negligible particle volume is then well satisfied. One important consequence of this assumption is that the equilibrium flow of the mixture of particles with a perfect gas can be analyzed like flow of a perfect gas that has the density and specific heat of mixture. Carrier (1958) was first to study the motion of Shock wave in dusty gases. Various aspects of two-phase flows were studied by Soo (1961). Kribel (1964) has analyzed normal shock-wave in particle laden gas. Rudinger (1964) has studied the some properties of shock relaxation in gas flows carrying small particles. Marble (1970) has discussed dynamics of dusty-gases, Mishra and Srivastava (1965) has studied the three dimensional shock wave in a dusty medium. Sharma et al. (1992) have discussed propagation of rapid pulses through a two-phase mixture of gas and dust particle, Pandey and Saxena (1989) has discussed growth and decay of sonic waves in two phase flows. Pandey and Singh (2011) have discussed non self similar solution of shock waves in gas particle mixture. They (2009) have also discussed strong explosions in two phase mixture of radiating gases.

At high gas densities (high pressure) or at high particle mass fractions, the particle volume fraction may become sufficiently large, so that it may be included into flow analysis without introducing significant errors. Since the particles may be considered as incompressible in comparison with the gas, the particle volume fraction enters into the basic flow equations as an additional variable. The interesting properties of such two phase flows is that even equilibrium flows cannot be treated as perfect gas flows. Rudinger (1969) has discussed different aspect of 
two-phase flows with and without particle volume fraction. Steiner and Hirschler (2002) have studied self similar solution of a shock propagation in a dusty gas. Jena and Sharma (1999), using Lie Group method have obtained entire class of self similar solution in dusty gases. Pandey and Vaish (2007) have discussed certain features of waves in two phase flows. Pandey and Saxena (1992) have discussed effects of finite volume fraction on curvature of a reflected shock in gas mixture. Pandey and Shukla (1998) have discussed the effect of radiative heat transfer on reflection of curved shock from straight rigid boundary through two phase mixture of gas and dust particle. There are many engineering problem in which dilute phase of gas particles is a good approximation of actual conditions. In such cases due to the existence of solid particles in the gas, properties of mixture differ significantly from those of gas alone. Such types of studies have numerous applications in underground explosion (Lamb, Collen, \& Sullivan, 1992; Nagayama, 1993).

\section{Basic Equations}

We consider the nozzle flow of gas particle mixture when cross sectional area of duct is not constant but a prescribed function $\mathrm{A}(\mathrm{x})$. We consider that the shock is to be produced by a piston moving with appropriate constant speed far back in the uniform section. The piston is still providing the thrust to keep the shock moving, but there are no changes due to this and the changes are entirely due to the cross-sectional area. Though the flow is not strictly one-dimensional but if the cross-section $\mathrm{A}(\mathrm{x})$ does not vary too rapidly the equation are obtained by averaging across the tube will provide a good approximation of one-dimensional flow. Bailey et al. (1961) have discussed gas particle flow in an axisymmetric nozzle. In this connection work done by Gilbert et al. (1962) and Kliegel (1963) is worth mentioning, as they have discussed dynamics of two phase flow in rocket nozzles and Gas particle nozzle flow respectively.

Equations governing one-dimensional motion of two phase flows when particle volume fraction is taken into account are given by Rudinger (1969),

$$
\begin{gathered}
A\left(\sigma+\sigma_{p}\right),_{t}+(\sigma u A),{ }_{x}+\left(\sigma_{p} u_{p} A\right),,_{x}=0, \\
\sigma\left(u,,_{t}+u u,_{x}\right)+\sigma_{p}\left(u_{p, t}+u u_{p, x}\right)+p_{, x}=0, \\
A\left[\sigma\left(\frac{u^{2}}{2}+C_{v} T\right)+\sigma_{p}\left(\frac{u_{p}^{2}}{2}+C T_{p}\right)\right]_{, t}+\left[\sigma u A\left(\frac{u^{2}}{2}+C_{p} T\right)+\sigma_{p} u_{p} A\left(\frac{u_{p}^{2}}{2}+C T_{p}+\varepsilon \frac{p}{\sigma_{p}}\right)\right]_{, x}=0, \\
A \varepsilon,_{t}+(A \varepsilon u),_{x}=0 .
\end{gathered}
$$

Equations (1-4) represents equation of mass, momentum, energy and conservation of particle mass fraction respectively. The symbols $\sigma, u, T, p, C_{p}, C_{v}$ denotes gas concentration, velocity, temperature, pressure, specific heat at constant pressure and at constant volume respectively. $\sigma_{p}, u_{p}, T_{p}, C, \varepsilon$ are particle concentration, velocity, temperature, specific heat of particle and particle volume fraction respectively and A being duct area.

The equation of state in present case can be written as,

$$
p=\frac{\rho_{m} R_{m} T}{1-\varepsilon},
$$

where $\rho_{m}=(1-\varepsilon)(1+\eta) \rho$ is density of mixture and $R_{m}=(1-\phi) R$ is effective gas constant for mixture, $\eta, \phi$ are mass flow ratio and mass flow rate respectively.

Using above equation of state Equations (1-4) can be rewritten in the following form,

$$
\begin{gathered}
(1-\varepsilon) \rho,_{t}+u(1-\varepsilon) \rho,_{x}+\rho u,_{x}+(\rho u / A)(1-\varepsilon) A,_{x}=0, \\
u,_{t}+u u,,_{x}+\{1 / \rho(1+\eta)(1-\varepsilon)\} p,,_{x}=0, \\
p,_{t}+u p,_{x}-a_{e}^{2}\left\{\rho,_{t}+u \rho,_{x}\right\}=0 \\
\varepsilon,_{t}+u \varepsilon,_{x}+\varepsilon u,_{x}+\{\varepsilon u / A\} A,_{x}=0
\end{gathered}
$$

where $a_{e}^{2}=\frac{p \gamma_{m}}{\rho_{m}(1-\varepsilon)}$ and $\gamma_{m}=\frac{\gamma(1+\eta \xi)}{(1+\gamma \eta \xi)}$ with $\xi=\frac{C}{C_{p}}$, are equilibrium sound speed and ratio of specific heats for mixture.

\section{Conservation Laws and Shock Relations}

Shock-Waves are the most important distinctive feature of supersonic flow, across which the medium undergoes sudden and often considerable changes in velocity, pressure, density and temperature. When the quantities (velocity, pressure, density and temperature) changes as we cross the surface it is called the surface of discontinuity 
or simply a shock. The occurrence of shock waves is commonly associated with supersonic flight, explosions and electric discharges and underground explosions. During study of such surface of discontinuity the equation of motion in front and behind are given by equations of motion given in section 2, but on surface of discontinuity these equation are not valid and to study the properties of such surfaces following conservation laws are used.

Conservation of mass, momentum and energy for two phase flows when particle volume fraction is taken as an additional variable are given by Rudinger (1969),

$$
\begin{gathered}
(1-\varepsilon) \rho u A=\left(1-\varepsilon_{0}\right) \rho_{0} u_{0} A=m, \\
\varepsilon \rho_{p} u_{p} A=\varepsilon_{0} \rho_{p} u_{0} A=\eta m, \\
m u+\eta m u_{p}+A p=m(1+\eta) u_{0}+A p_{0}, \\
\frac{u^{2}}{2}+C_{p} T+\eta\left\{\frac{u_{p}^{2}}{2}+C T_{p}+\frac{p}{\rho_{p}}\right\}=(1+\eta) \frac{u_{0}^{2}}{2}+\left(C_{p}+\eta C\right) T_{0}+\eta \frac{p_{0}}{\rho_{p}} .
\end{gathered}
$$

When equilibrium is eventually established, we can write $u=u_{p}=u_{e}$ and thus Equations (9-12) reduces in to following set of equations,

$$
\begin{aligned}
\left(1-\varepsilon_{e}\right) \rho_{e} u_{e} A & =\left(1-\varepsilon_{0}\right) \rho_{0} u_{0} A=m, \\
\varepsilon_{e} u_{e} A & =\varepsilon_{0} u_{0} A=\eta m, \\
m(1+\eta) u_{e}+A p_{e} & =m(1+\eta) u_{0}+A p_{0}, \\
(1+\eta) \frac{u_{e}^{2}}{2}+\left(C_{p}+\eta C\right) T_{e}+\eta \frac{p_{e}}{\rho_{e}} & =(1+\eta) \frac{u_{0}^{2}}{2}+\left(C_{p}+\eta C\right) T_{0}+\eta \frac{p_{0}}{\rho_{0}},
\end{aligned}
$$

where subscripts e and 0 denotes the quantities in equilibrium and initial states respectively. Solving Equations (13-16) we have following shock conditions,

$$
\begin{gathered}
\frac{u_{e}}{u_{0}}=\frac{\left(\gamma_{m}-1\right) M_{e}^{2}+2+2 \varepsilon_{0}\left(M_{e}^{2}-1\right)}{\left(\gamma_{m}+1\right) M_{e}^{2}}, \\
\frac{\varepsilon_{e}}{\varepsilon_{0}}=\frac{u_{o}}{u_{e}}, \\
\frac{\rho_{e}}{\rho_{0}}=\left\{\frac{\left(1-\varepsilon_{0}\right)}{\left(1-\varepsilon_{e}\right)}\right\} \frac{u_{0}}{u_{e}}, \\
\frac{p_{e}}{p_{0}}=1+\frac{\gamma u_{0}\left(u_{0}-u_{e}\right)(1+\eta)\left(1-\varepsilon_{o}\right)}{a_{0}^{2}}=1+\frac{2 \gamma_{m}\left(M_{e}^{2}-1\right)}{\left(\gamma_{m}+1\right)} .
\end{gathered}
$$

Equations (17-20) can be written in following form also,

$$
\begin{gathered}
u_{e}=\frac{a_{o} M_{0}\left\{\left(\gamma_{m}-1\right) M_{e}^{2}+2+2 \varepsilon_{0}\left(M_{e}^{2}-1\right)\right\}}{\left(\gamma_{m}+1\right) M_{e}^{2}}, \\
\varepsilon_{e}=\frac{\varepsilon_{o}\left(\gamma_{m}+1\right) M_{e}^{2}}{\left\{\left(\gamma_{m}-1\right) M_{e}^{2}+2+2 \varepsilon_{0}\left(M_{e}^{2}-1\right)\right\}}, \\
\rho_{e}=\frac{\rho_{o}\left(1-\varepsilon_{0}\right)\left(\gamma_{m}+1\right) M_{e}^{2}}{\left(1-\varepsilon_{e}\right)\left\{\left(\gamma_{m}-1\right) M_{e}^{2}+2+2 \varepsilon_{0}\left(M_{e}^{2}-1\right)\right\}}, \\
p_{e}=a_{o}^{2} \rho_{0}\left[\frac{2 M_{e}^{2}}{\left(\gamma_{m}+1\right)}-\frac{\left(\gamma_{m}-1\right)}{\gamma_{m}\left(\gamma_{m}+1\right)}\right],
\end{gathered}
$$

where $M_{e}$ is Mach number defined as $M_{e}^{2}=\frac{u_{e}^{2}}{a^{2}}, u$ is velocity of medium and $a$ is speed of sound.

With help of Equations (21-24) and taking $M_{e} \rightarrow \infty$ we have following strong shock conditions,

$$
u_{e}=u_{0}\left(\frac{\gamma_{m}-1+2 \varepsilon_{0}}{\gamma_{m}+1}\right) \text {, }
$$




$$
\begin{gathered}
\varepsilon_{e}=\varepsilon_{0}\left(\frac{\gamma_{m}+1}{\gamma_{m}-1+2 \varepsilon_{0}}\right), \\
\rho_{e}=\rho_{0}\left(\left(\frac{1-\varepsilon_{0}}{1-\varepsilon_{0}}\right) \frac{\gamma_{m}+1}{\gamma_{m}-1+2 \varepsilon_{0}}\right), \\
p_{e}=\rho_{0} u_{e}^{2}\left(\frac{2}{\gamma_{m}+1}\right) .
\end{gathered}
$$

\section{Shock Propagation Down a Non-Uniform Tube}

A detail investigation of propagation of shock-wave along ducts of varying cross-section is given by Chester (1954). In this section using Whitham's rule (Whitham, 1974) of characteristics propagation down a non-uniform tube of variable cross sectional area is discussed. Vishwakama (2002) has applied Whitham's rule (Whitham, 1974) of characteristics for imploding cylindrical shock waves under the action of a magnetic field. In this connection work done by Sharma and Madhumita (2004) is also worth mention.

Applying Whitham's rule (Whitham, 1974) of characteristic equations for Equations (5-7) are given as follows ,

$$
\begin{gathered}
\left\{\frac{\partial}{\partial t}+\left(u_{e}+a_{e}\right) \frac{\partial}{\partial x}\right\}\left\{p+u a_{e} \rho_{e}\left(1-\varepsilon_{e}\right)(1+\eta)\right\}+\frac{u_{e} a_{e}^{2} \rho_{e}}{A} A, x_{x}=0, \\
\left\{\frac{\partial}{\partial t}+\left(u_{e}-a_{e}\right) \frac{\partial}{\partial x}\right\}\left\{p-u a_{e} \rho_{e}\left(1-\varepsilon_{e}\right)(1+\eta)\right\}+\frac{u_{e} a_{e}^{2} \rho_{e}}{A} A,,_{x}=0, \\
\left\{\frac{\partial}{\partial t}+u_{e} \frac{\partial}{\partial x}\right\}\left\{p-a_{e}^{2} \rho\right\}=0,
\end{gathered}
$$

where Equations (25) and (26) corresponds to the equations along the characteristics $\frac{d x}{d t}=u_{e} \pm a_{e}$ respectively. Integrating above equations along characteristic the general solution of above equations are given by,

$$
\begin{gathered}
\left\{p_{e}-p_{0}\right\}+a_{e} \rho_{e}\left(1-\varepsilon_{e}\right)(1+\eta)\left(u_{e}-u_{o}\right)=-\frac{u_{e} a_{e}^{2} \rho_{e}}{\left(u_{e}+a_{e}\right)}\left(\frac{A(x)-A_{o}}{A_{o}}\right)+F\left\{x-\left(u_{e}+a_{e}\right) t\right\}, \\
\left\{p_{e}-p_{0}\right\}-a_{e} \rho_{e}\left(1-\varepsilon_{e}\right)(1+\eta)\left(u_{e}-u_{o}\right)=-\frac{u_{e} a_{e}^{2} \rho_{e}}{\left(u_{e}+a_{e}\right)}\left(\frac{A(x)-A_{o}}{A_{o}}\right)+G\left\{x-\left(u_{e}-a_{e}\right) t\right\}, \\
\left\{p_{e}-p_{0}\right\}-a_{e}^{2}\left(\rho_{e}-\rho_{o}\right)=H\left\{x-u_{e} t\right\},
\end{gathered}
$$

where $F, G$ and $H$ are arbitrary functions. These solutions were obtained by Whitham (1974) and we have extended it for two-phase flow which are applicable for blood flow in arteries, water flow in pipes and explosions in stars. In terms of the change in Mach number $\left(M_{e}-M_{0}\right)$ perturbations $\left(p_{e}-p_{0}\right),\left(u_{e}-u_{0}\right)$, are given by,

$$
\begin{gathered}
p_{e}-p_{0}=\frac{4 a_{o}^{2} \rho_{0} M_{e}}{\left(\gamma_{m}+1\right)}\left(M_{e}-M_{0}\right), \\
u_{e}-u_{0}=\frac{2 a_{0}}{\left(\gamma_{m}+1\right)}\left(1+\frac{1}{M_{0}^{2}}\right)\left(M_{e}-M_{0}\right)\left(1-\varepsilon_{0}\right), .
\end{gathered}
$$

When these are substituted in Equation (28) with $(F=0)$, we have,

$$
\left[\frac{4 M_{o}}{\left(\gamma_{m}+1\right)}+\frac{2}{\left(\gamma_{m}+1\right)}\left(1+\frac{1}{M_{0}^{2}}\right)\left(1-\varepsilon_{0}\right) \frac{\rho_{e} a_{e}}{\rho_{0} a_{0}}\right]\left(M_{e}-M_{0}\right)=-\frac{u_{e} a_{e}^{2} \rho_{e}}{a_{0}^{2} \rho_{0}\left(u_{e}+a_{e}\right)}\left(\frac{A(x)-A_{o}}{A_{o}}\right),
$$

The expressions for $u_{e}, \rho_{e}$ in terms of $M_{0}$ are given by Equations (21) and (23) with $M_{e}=M_{0}$. Equation (33) after certain manipulation becomes,

$$
\left(\frac{A(x)-A_{o}}{A_{o}}\right)=-g\left(M_{0}\right)\left(M_{e}-M_{0}\right)
$$

where,

$$
g\left(M_{e}\right)=\frac{M_{e}}{\left(M_{e}^{2}-1\right)}\left[1+\frac{2\left(1-\varepsilon_{0}\right)}{\left(\gamma_{m}+1\right)} \frac{\left(1-\mu^{2}\right)}{\mu}\right]\left[1+2 \mu+\frac{1}{M_{e}^{2}}\right]
$$


and

$$
\mu^{2}=\frac{\left\{\left(\gamma_{m}-1\right) M_{e}^{2}+2+2 \varepsilon_{0}\left(M_{e}^{2}-1\right)\right\}}{2 \gamma_{m} M_{e}^{2}-\left(\gamma_{m}-1\right)} .
$$

Now we are applying Whitham's rule (1974) of characteristic to find change in area $A(x)$ along characteristic $\frac{d x}{d t}=u_{e}+a_{e}$.

For a tube which varies slowly but which accumulates large changes in $A(x)$ over a sufficiently large length, we might break down the problem into successive small lengths in each of which the change in $A(x)$ is small. In each such small length of tube it would be admissible to linearize about the local condition and develop a small perturbation theory as in Equations (31) to (34). But it would no longer be strictly valid to take $F=0$, because the entry conditions into each of these subsections would not be a uniform state. As Equation (34) is the differential form of a functional relation $M=M(A)$,

$$
\frac{1}{A} \frac{d A}{d M}=-g(M)
$$

The characteristic equation for the $\frac{d x}{d t}=u+a$ characteristic in this case is,

$$
\frac{d p}{d x}+\rho(1-\varepsilon)(1+\eta) a \frac{d u}{d x}+\frac{\rho a^{2} u}{(u+a)} \frac{1}{A} \frac{d A}{d x}=0 .
$$

Substituting shock conditions given as in Equations (21-24), we have,

$$
g(M) \frac{d M}{d x}+\frac{1}{A} \frac{d A}{d x}=0,
$$

where $g(M)$ is given by Equation (35). We can write Equation (39) as,

$$
\frac{M}{M^{2}-1} \lambda(M) \frac{d M}{d x}+\frac{1}{A} \frac{d A}{d x}=0,
$$

where,

$$
\lambda(M)=\left[1+\frac{2\left(1-\varepsilon_{0}\right)}{\left(\gamma_{m}+1\right)} \frac{\left(1-\mu^{2}\right)}{\mu}\right]\left[1+2 \mu+\frac{1}{M_{0}^{2}}\right] .
$$

Equation (40) can be written as in the form,

$$
\frac{1}{A} d A=\frac{-M}{M^{2}-1} \lambda(M) d M
$$

If we consider the case when $M \rightarrow 1, \lambda \rightarrow 4$ which shows that particle does not contribute to this case but strong shock case when $M \rightarrow \infty, \mu^{2}=\frac{\left\{\left(\gamma_{m}-1\right)+2 \varepsilon_{0}\right\}}{2 \gamma_{m}}$, hence presence of particle effect $\mu^{2}$ and hence $\lambda$ also.

\section{Result and Discussion}

For various values of particle volume fraction $(\varepsilon=0,0.05,0.15,0.25)$ velocity, density, pressure are obtained on shock surface from Equations (17-20) and they are in agreement with well known results. Figure 1 shows that the velocity decreases behind the shock waves a well known result but presence of particle volume fraction shows that it is large as compared to pure gas case. Figure 3 shows that well known result conservation of mass is satisfied and density increases behind the shock wave but it always less than to the pure gas case and as value of particle volume fraction is increasing density is decreasing. Figure 5 shows variation of pressure behind the shock wave for air, dry and wet stream which shows that pressure has increasing tendency but for dry and wet stream it is less than that of air. From Equations (21) and (23) velocity and density are obtained for various values of equilibrium Mach number $\left(M_{e}=2.85,3.85,4.85\right)$. The nature of velocity and density are shown through Figures 2 and 4 . With help of Equation (41) variation of area is obtained and plotted for equilibrium Mach number $\left(M_{e}=2.85,3.85,4.85\right)$ in Figure 6. For strong shock case $M \rightarrow \infty, \mu^{2}=\frac{\left\{\left(\gamma_{m}-1\right)+2 \varepsilon_{0}\right\}}{2 \gamma_{m}}$, which shows the effect of particle volume fraction on $\mu^{2}$ and hence on $\lambda$ also. Variation of $\lambda$ for different values of $\varepsilon$ is shown in Figure 7 which shows that as particle volume faction is increasing $\lambda$ is decreasing. 


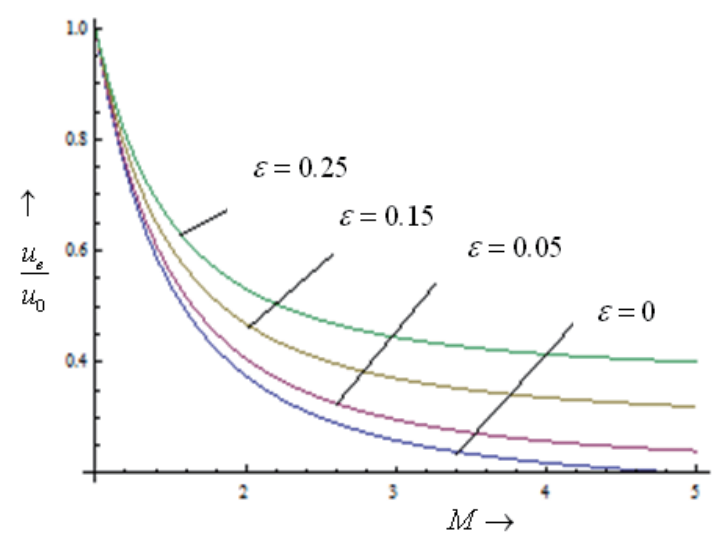

Figure 1. A graph between Mach number ' $M$ ' and ratio of velocity ' $\left(u_{e} / u_{0}\right)$ ' for different values of particle volume fraction ' $\varepsilon$ '

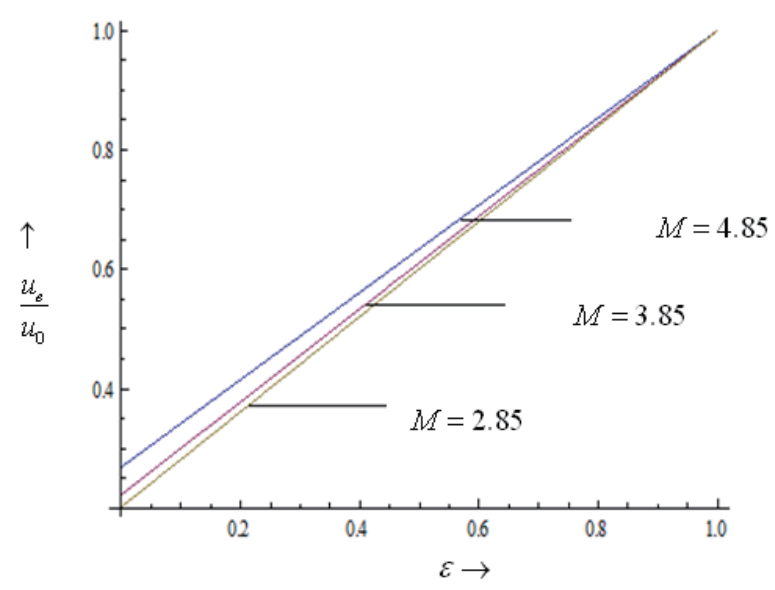

Figure 2. A graph between the particle volume fraction ' $\varepsilon$ ' and ratio of velocity ' $\left(u_{e} / u_{0}\right)$ ' for different values of Mach number ' $M$ '

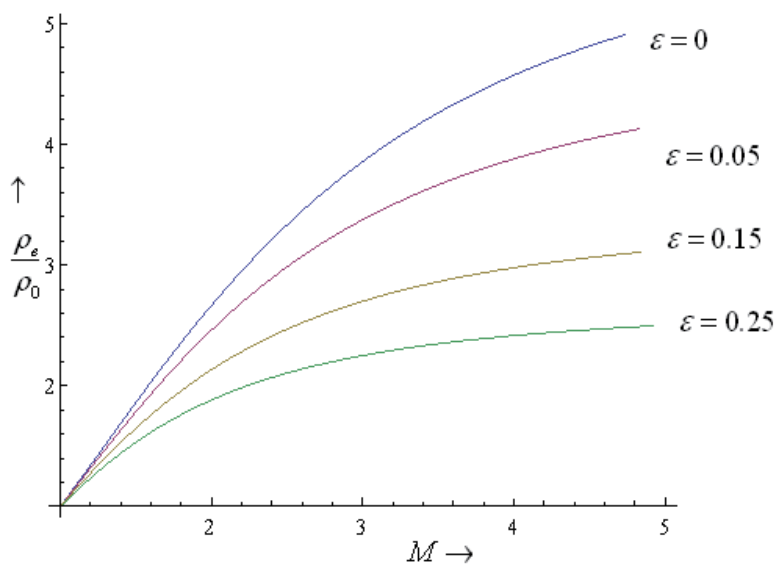

Figure 3. A graph between the Mach number ' $M$ ' and ratio of density ' $\left(\rho_{e} / \rho_{0}\right)$ ' for different values of particle volume fraction ' $\varepsilon$ ' 


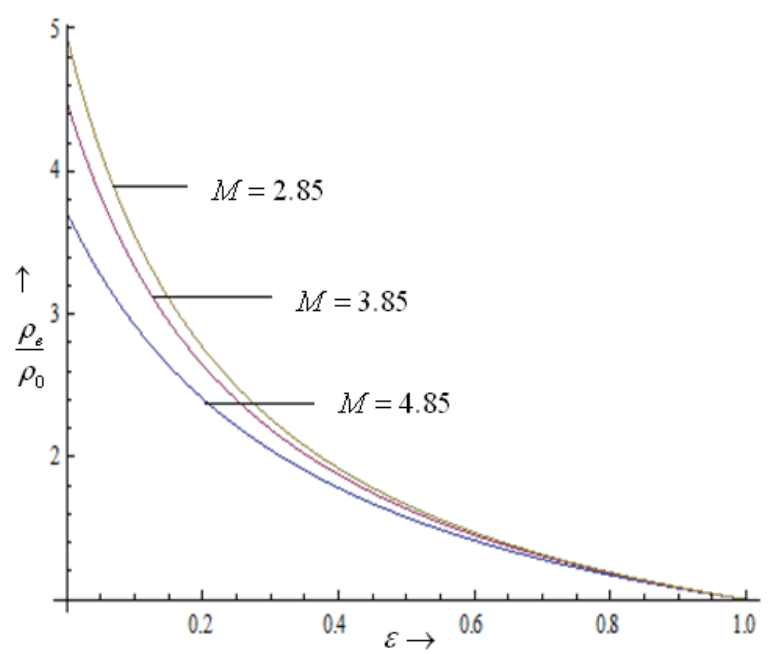

Figure 4. A graph between the particle volume fraction ' $\varepsilon$ ' and ratio of density ' $\left(\rho_{e} / \rho_{0}\right)$ ' for different values of Mach number ' $M$ '

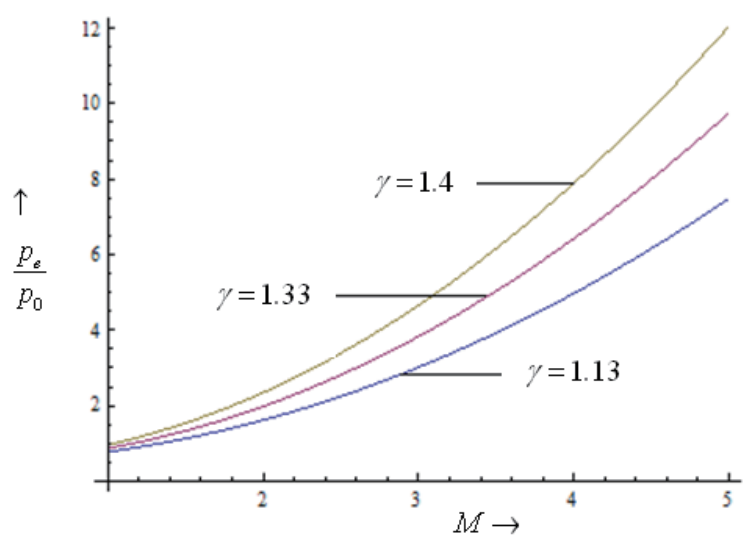

Figure 5. A graph between the Mach number ' $M$ ' and ratio of pressure ' $\left(p_{e} / p_{0}\right)$ ' for different values of particle volume fraction ' $\varepsilon$ '

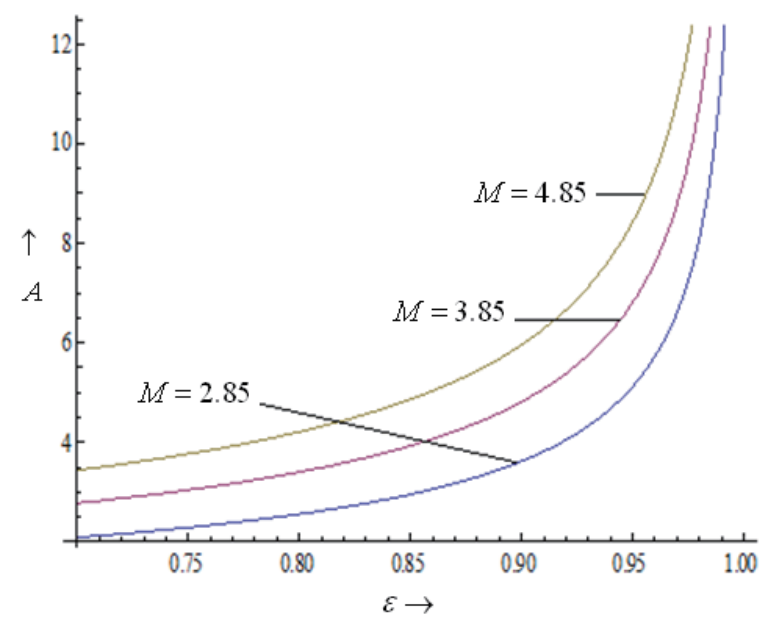

Figure 6. A graph between the particle volume fraction ' $\varepsilon$ ' and area ' $A$ ' for different values of Mach number ' $M$ ' 


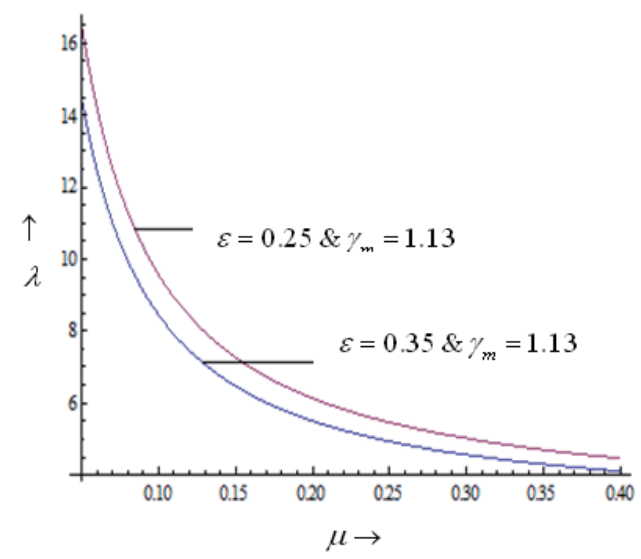

Figure 7. A graph between ' $\lambda$ ' and shock relative to the flow ' $\mu$ ' for different values of particle volume fraction ' $\varepsilon$ '

\section{Acknowledgements}

Authors are grateful to the refries for their valuable suggestions and one of the author Kanti Pandey is grateful to U.G.C. for providing financial assistance in preparation of this article.

\section{References}

Bailey, W. S., \& Serra, R. A. (1961). Gas particle flow in an axisymmetric nozzle. ARSJ, 31, 793-799. http://dx.doi.org/10.2514/8.5636

Carrier, G. F. (1958). Shock waves in dusty gas. J. of Fluid Mech., 4, 376-382. http://dx.doi.org/10.1017/S0022112058000513

Chester, W. (1954). The quasi-cylindrical shock tube-Phil. Mag., 45(7), 1293-1301.

Gilbert, M., Allport, J., \& Dunlop, R. (1962). Dynamics of two phase flow in rocket nozzles. ARSJ., 32(12), 1929-1930.

Jena, J., \& Sharma, V. D. (1999). Self Similar Shocks in a Dusty Gas. International J. of Non-linear Mechanics, 34, 313-327. http://dx.doi.org/10.1016/S0020-7462(98)00035-3

Kliegel, J. R. (1963). Gas particle nozzle flow. Symp. Combust., 9(1), 811-826. http://dx.doi.org/10.1016/S0082-0784(63)80088-0

Kriebel, A. R. (1964). Analysis of normal shock waves in particle laden gas. J. Basic Eng., 86, 655-665. http://dx.doi.org/10.1115/1.3655914

Lamb, F. K., Collen, B. W., \& Sullivan, J. D. (1992). An approximate analytical model of shock waves from underground nuclear explosion. J. Geophys. Res. $B_{1}, 97,515-535$.

Marbel, F. E. (1970). Dynamics of dusty gases. Ann. Rev. Fluid Mech., 2, 397-446. http://dx.doi.org/10.1146/annurev.fl.02.010170.002145

Mishra, R. S., \& Srivastava, O. S. (1965). Three dimensional shock waves in a dusty medium. Tensor N. S., 16(2), $133-142$.

Nagayama, K. (1993). Shock wave interaction in solid material. In A. B. Sawaoka (Ed.), Shock waves in material science. Berlin: Springer. http://dx.doi.org/10.1007/978-4-431-68240-0_9

Pandey, K., \& Saxena, M. (1989). Growth and decay of sonic waves in two phase flow. Prog. Of Maths, 23 (1 and 2, pp. 125-135).

Pandey, K., \& Saxena, M. (1992). Effects of finite volume fraction on curvature of a reflected shock in gas mixture. Jour. Math. Phy. Sci., 26(1), 61-76.

Pandey, K., \& Shukla, M. K. (1998). Effect of Radiative Heat Transfer on Reflection of Curved Shock From Straight Rigid Boundary Through two Phase Mixture of Gas and Dust Particle. Proc. Nat. Sci. India, 68(II), $135-150$. 
Pandey, K., \& Singh, K. (2009). Strong explosions in two phase mixture of radiating gases. J. of Int. Academy of Physical Sciences, 13(3), 217-229.

Pandey, K., \& Singh, K. (2011). Non self similar solution of shock waves in gas particle mixture. Rev. Bull. Cal. Math. Soc., 19(1), 69-82.

Pandey, K., \& Vaish, A. K. (2007). Waves in two Phase Flows. Advanced Studies in Pure Mathematics, 47(1), 303-320.

Rudinger, G. (1964). Some properties of shock relaxation in gas flows carrying small particles. Phys. of Fluid, 7 , 658-663. http://dx.doi.org/10.1063/1.1711265

Rudinger, G. (1969). In P. P. Wegener (Ed.), Relaxation in gas particle flow-Non Equilibrium Flows (Part 1, pp. 119-159). New York and London: Marcel Dekker.

Sharma, V. D., \& Gupta, N. (1992). Propagation of rapid pulses through a two phase mixture of gas and dust particle. Int. J. Eng. Sci., 30, 263-272. http://dx.doi.org/10.1016/0020-7225(92)90071-N

Sharma, V. D., \& Madhumita, G. (2004). Imploding cylindrical and spherical shock waves in a non ideal medium. Jour. of Hyperbolic Differential Equations, 1(3), 521-530. http://dx.doi.org/10.1142/S0219891604000184

Soo, S. L. (1961). Gas dynamic processes involving suspended solids. A. I. Ch. E. J., 7(3), 384-391. http://dx.doi.org/10.1002/aic.690070308

Steiner, H., \& Hirschler, T. (2002). A Self Similar Solution of a Shock Propagation in a Dusty Gas. Eur. J. Mech. B/Fluids, 21(3), 371-380. http://dx.doi.org/10.1016/S0997-7546(02)01181-0

Vishwakarma, J. P. (2002). Imploding cylindrical shock waves under the action of a magnetic field. In P. Manchanda, K. Ahemd, \& A. H. Siddiqui (Eds.), Current trends in industrial and applied mathematics (pp. 109-122). New Delhi, India: Anamaya Publisher.

Whitham, G. B. (1974). Linear and Nonlinear Waves. New York: John Wiley \& Sons.

\section{Copyrights}

Copyright for this article is retained by the author(s), with first publication rights granted to the journal.

This is an open-access article distributed under the terms and conditions of the Creative Commons Attribution license (http://creativecommons.org/licenses/by/3.0/). 